\section{PHYSIOLOGY OF THE AMINO ACIDS*}

\author{
By DR. DONALD D. VAN SLYKE \\ Rockefeller Institute for Medical Research
}

$\mathrm{T}$ $\mathrm{HE}$ number of proteins in the animal and vegetable world appears to be infinite. Yet they are all constructed of a relatively few units, namely, the amino acids. These amino acids in number are about equal to the letters of the alphabet. They have an extraordinary facility for being joined in chains; each amino acid has the same head and tail, so that the tail of one can link with the head of another, and the linking can be repeated apparently times without limit. Each amino acid has an $\mathrm{H}_{2} \mathrm{~N}$, or amino group, which has an alkalinity 'about equal to that of ammonia; and each has a $\mathrm{COOH}$ or carboxyl group, which has an acidity about like that of eitric acid. There is also a chemical group, $R$, which is different in each amino acid, and gives it its character as an individual. Hence we may call the $R$ group the individualizing group of each amino acid. The amino and carboxyl groups neutralize each other, so that if the individualizing group is neutral the amino acid is neutral; such are alanine, glycine, leucine. However, if the individualizing group is alkaline the amino acid is alkaline; such are lysine, arginine, and histidine.

Aside from its acid or basic properties, each individualizing group bears other chemical properties. In serine, threonine, and hydroxylysine, for example, the individualizing group bears a hydroxyl with all the reacting powers of an alcoholic hydroxyl. Again, the individualizing group may carry a second carboxyl; then the amino acid is strongly acidic: such are aspartic and glutamic acids.

In cystine and methionine the individualizing group has a sulphur atom. The cystine sulphur can take up a hydrogen atom to form cysteine, and when this occurs to cystine in a protein molecule the entire character of the protein is changed. In wool and hair the sulphur is in the oxidized .C-S-S.C. form of cystine, and the proteins are utterly insoluble and indigestible. If the -C.S.S.C- is reduced to two -C-SH groups, forming cysteine, the wool is changed into proteins : which are easily digested.

In proteins Emil Fischer demonstrated that the amino acids are joined, head to tail, by what he termed peptide linkings, each $\mathrm{NH}_{2}$ group condensing with the $\mathrm{COOH}$ of another amino acid, with elimination of the elements of water. Simple chains of a few amino acids Fischer termed peptides. The dipeptide glycolalanine still has a free $\mathrm{NH}_{2}$ group and a free COOH, where two more amino acids can be linked on, and the process can be repeated ad infinitum.

The proteins are peptides of tremendously long chains. These chains seem usually to be rolled into balls, like balls of yarn, or otherwise made to take globular or ellipsoid shape, for their rates of diffusion were found by Northrop and Kunitz to approximate to those calculated for spheres. Exceptions are the fibrous proteins, silk and wool, of which the molecules themselves appear to be extended into fibres.

In the human stomach the protein molecules meet the pepsin of the gastric juice, which unrolls the balls and breaks the overgrown chains into shorter ones, which are still fairly long, and go under the general

* Substance of a paper read at the Fifteenth Anniversary Celebrations of the University of Chicago on September 23, 1941. name of 'peptones'. The chief visible change in the stomach, as noted a century ago by Beaumont in the stomach of Alexis St. Martin, is that proteins which enter the stomach as insoluble matter, such as meat or coagulated egg white, leave the stomach and enter the intestine largely changed to soluble material.

We may pause here to point out one fallacy that has crept into the practice of medicine through a perfectly correct ohservation by Beaumont. He noted the times necessary for various foods to remain in the stomach before they were turned into soluble material and passed on into the intestine. He found that raw egg albumin was one of the quickest protein foods to pass through. From this it has been concluded that raw egg white is one of the most easily digested proteins. The fact is that it is one of the most resistant to digestion. It passes quickly and easily through the stomach because it happens to be soluble, and it reaches the intestine largely in its original undigested state. The digestive reserves of the intestine are so great that they normally complete the work of breaking down the egg protein chains. However, the egg protein is one of those which are most likely to be absorbed in the undigested state, and to cause sensitization, particularly in infants. In fact it is said that raw egg white fed to an infant has been observed to be absorbed in such amounts that it reappeared in the urine. The most digestible form in which egg proteins can be fed is that obtained by coagulating them with thorough cooking, and then breaking the material into finely divided particles by passage through a sieve.

In the intestine the gastric products meet the enzymes secreted by the pancreas and the intestinal wall. It used to be thought that the trypsin of the pancreas had the power to break at least part of the peptide chains completely to amino acids, but it now appears that trypsin can carry the digestion no further than to the stage of peptides of the average size of six or seven amino acids per peptide molecule.

Dr. Robert Dillon and I succeeded some years ago in devising a method of analysis which was specific for free amino acids. It depended on the ability of a mild oxidizing agent called ninhydrin, which first attacked the amino group of the amino acid, breaking off $\mathrm{NH}_{3}$ from the $R . \mathrm{CH}\left(\mathrm{NH}_{2}\right) \cdot \mathrm{COOH}$, and leaving in its place the keto acid R.CO.COOH. Boiling decomposes the keto acid into aldehyde and carbon dioxide. The actual analysis consists merely in boiling the amino acid and ninhydrin together and measuring the carbon dioxide evolved.

For the series of reactions that end in the evolution of carbon dioxide it is apparent that one must have both an $\mathrm{NH}_{2}$ group and a COOH group in adjacent positions, and this is the definition of an alpha-amino acid. If either the $\mathrm{NH}_{2}$ or the COOH group is tied up in a peptide linking, the reaction cannot go through to a finish and no carbon dioxide is evolved.

With this method Dr. MacFadyen, who followed Dillon in our laboratory, was able to show that purified crystalline trypsin splits protein to peptides, but not to free amino acids, but that 'crude trypsin', comprising a mixture of the pancreatic enzymes, goes much further and splits many of the peptides into free amino acids. It is evident that the pancreas produces peptidases as well as trypsin, but that pure trypsin is not a peptidase with the power to accom. plish the last step of protein hydrolysis, namely, to set the amino acids free.

Although the trypsin alone does not carry digestion to the stage of free amino acids, experiments both 
in vitro and in vivo have shown that the mixture of pancreatic and intestinal enzymes does produce free amino acids. Whether all the protein products in the intestine are digested to free amino acids before they are absorbed is not certain, but it does appear that the greater part of these products are absorbed as free amino acids. The evidence of this is that during protein digestion there is an increase in the free amino acid content of the mesenteric blood, and also of the blood of the general circulation, but reliable methods have failed to demonstrate an increase in peptide nitrogen, of which very little is present.

The speed with which digestion begins was evidenced by experiments of Cullen and McLean in which dogs were fed meat, and the blood was analysed for urea at short intervals. Within fifteen minutes the blood urea content had risen 10 per cent, indicating that a measurable part of the protein had already passed through all the stages, not only of digestion, but also of absorption and degradation. The process once started goes on steadily until absorption of the ingested protein is completed. The length of time required for completion varies in proportion to the amount ingested, but is usually $2-4$ hours.

To find where the absorbed amino acids were taken from the blood, and what happened to them, Cullen and MeLean anæsthetized dogs during digestion and quickly drew blood from the portal vein between intestine and liver, from the mesenteric vein leaving the liver, and from the vena cava, where blood from all the tissues is mixed. It was found that the amino acid content of the blood rose about 20 per cent as the blood perfused the intestines, and that the greater part of the absorbed amino acids were removed by the liver. In return, the liver poured into the blood of the hepatic vein an amount of urea nitrogen which almost balanced the amino acid nitrogen that had been taken up. One could watch the work of the liver in taking up the amino acids and destroying them, turning their nitrogenous parts into urea for excretion by the kidneys.

Other experiments, performed with Dr. Gustav Meyer, showed that the liver did not get quite all the absorbed amino acids, but that some escaped, and could be absorbed by other tissues. In order to magnify the effects of such absorption blood amino acid concentrations were raised to high levels by intravenous injection of amino acid solutions. Portions of muscle, kidney, and liver tissue were analysed for amino nitrogen before and after the injections. It was found that even in the fasting animal the amino acid concentration in the tissues was about eight times as great as in the blood plasma, namely, about $40 \mathrm{mgm}$. of amino acid nitrogen per $100 \mathrm{gm}$. of tissue, compared with $5 \mathrm{mgm}$. per $100 \mathrm{gm}$. of plasma. When amino acids were injected the amounts taken up by the glandular organs were rnuch greater than by the muscles. The content of the muscles in amino acid nitrogen rose to about $60 \mathrm{mgm}$., of the kidneys to about $80 \mathrm{mgm}$., and of the liver to about 120 mgm. per $100 \mathrm{gm}$. of tissue. During the next three hours the amino acids in the muscles and kidneys remained practically unchanged, but the amino acids in the liver almost fell back to their original level, and an equivalent of urea nitrogen appeared in the circulation.

The evidence in these experiments, that the liver is the organ where urea formation takes place, supported an ancient but much contested hypothesis that the liver is the only organ that forms urea. Its unique distinction in this power was clinched by
Bollman, Mann, and Magath, of the Mayo Clinic, who showed that removal of the livers from dogs led to an accumulation of amino acids in the blood, and entirely stopped the formation of urea.

The chemical steps by which the nitrogen of the amino acids is converted by the liver into urea have been demonstrated by Krebs. The nitrogen is first oxidized off as ammonia. Two molecules of ammonia then combine with one of the diamino acid, ornithine, to form the 4-nitrogen amino acid arginine. The arginine is then hydrolysed by the enzyme arginase in the liver, with formation of one molecule each of urea and ornithine. The net result is to change the highly toxic ammonia into the non-toxic and highly diffusible urea, an end-product ideally suited for transportation to the kidneys and excretion. The ornithine can go through the cycle an indefinite number of times, taking up two molecules of ammonia and giving them off as one molecule of urea. The ornithine thus acts as a true catalyst. Liver had been demonstrated years before by Dakin to be rich in the enzyme arginase, which hydrolyses arginine to urea and ornithine, but the physiological purpose of the arginase in the liver had been a mystery until Krebs' work showed its role in the final step of urea formation.

Another vicissitude of the amino acids which the work of Mann and his colleagues located in the liver is the transformation into glucose. Graham Lusk, in the early part of the century, showed that when protein is catabolized by dogs made totally diabetic by phloridzin poisoning, about $60 \mathrm{gm}$. of glucose was formed and excreted from each $100 \mathrm{gm}$. of protein catabolized. Lusk and his collaborators also showed that certain of the amino acids had their carbon partly or entirely turned into glucose when their nitrogen was turned into urea. Mann and his colleagues showed that no glucose formation from proteins or amino acids occurred when the liver was excluded.

Furthermore, the acceleration of the body's heat production that occurs during assimilation of protein digestion products was shown by Mann and his colleagues not to occur when the liver was excluded. This accelerated heat production, called by Lusk the "specific dynamic action", apparently either represents energy produced by the reactions which the amino acids undergo in the liver, or is caused by other reactions in the cells which are stimulated by the presence of products formed in the liver. Such substances must be other than the urea and glucose, for neither of these shows the observed amount of stimulating effect. The specific dynamic effect may be due either to heat evolved from reactions that occur in the liver, or to the effect of products, other than urea or glucose, in stimulating other heat-producing reactions in the body. The decision between these two possibilities awaits future exploration.

Not all the treatment met by the amino acids in the liver is destructive. During periods of heavy protein feeding the body stores considerable amounts of protein in the liver and to a less degree in the other tissues. The protein seems to be different from the structural proteins of the tissues. In the liver Berg has shown that it can, in fact, be differentiated with the microscope by its droplet structure in the cells. Functionally it is characterized by the readiness with which it is metabolized when the protein intake is decreased, or with which it is used to replace blood proteins depleted by hæmorrhage, as found by Whipple and his colleagues. It appears probable that this protein is constructed in the liver part of the amino acids brought to it from the intestine. 
Besides the liver, all the living tissues of the body take their shares in the metabolism of the amino acids, for all of them are of substance chiefly protein, and it is a living protein that is continually being broken down and rebuilt from its constituent amino acids. A most interesting part of this process has recently been discovered by two Russian biochemists named Braunstein and Kritzman. It is called 'transamination', and it enables the cells to change keto acids to amino acids by replacing the oxygen atom of the ketone with ammonia, transferred to the $\mathrm{CO}$ group of the ketone acid from the $\mathrm{CH}\left(\mathrm{NH}_{2}\right)$ of either of the dicarboxylic amino acids, aspartic and glutamic. Since at least some of the keto acids can be produced by partial oxidation of carbohydrates, transamination appears to be one of the processes hy which the body can construct a portion of its own amino acids; and it has been well established that the body can in fact synthesize about half the amino acids that are found in its protein structures. The reaction of transamination is caused by an enzyme that occurs in all the tissues, but is particularly rich in the muscles.

It is a peculiar thing that only the acidic amino acids, glutamic and aspartic, with two carboxyl groups each, can give their nitrogen to keto acids. Monocarboxylic amino acids, such as glycine and alanine, cannot transfer their nitrogen to keto acids to and from each other. However, they can transfer their nitrogen to keto-succinic and keto-glutamic acids to form aspartic and glutamic acids; this is a reversible process. With aspartic or glutamic acids as an intermediary step, therefore, it seems to be possible for leucine, for example, to transfer its amino group to pyruvic acid and make alanine, or in reverse, for alanine to donate its nitrogen to make leucine from its keto acid. In either case the shifting nitrogen first is transferred to glutamic as aspartic acid, and then from that to the keto acid. The transfer is affected by an enzyme, which one of its investigators, Cohen, suitably calls transaminase; it has been separated in the form of a protein from tissue extracts and exerts its activity in the absence of living cells.

From the fact that by the transaminase action the body can build up at least some of its own amino acids it would follow that not all the amino acids in the body proteins need be provided ready-made in the food proteins, but that some can be made in the body provided the necessary products to form the keto acids are available. In fact, thirty-four years ago Magnus-Levy demonstrated that the rabbit can make the simplest amino acid, glycine. When benzoic acid is fed to an animal it is detoxified by conjugating it with glycine to form hippuric acid, which is excreted in the urine. Magnus-Levy, by feeding hippuric acid to rabbits on nitrogen-free diets, was able to make them excrete as hippuric acid more glycine than they had contained in their entire bodies. They had used other nitrogenous materials to build glycine in order to detoxify the hippuric acid. The task of finding which of the other twenty amino acids are indispensable parts of the diet and which one the animal body can build for itself has occupied some of the leading biochemists since the time of Magnus-Levy's demonstration. The names of F. Gowland Hopkins in Great Britain and of Lafayette Mendel, T. B. Osborne, and W. C. Rose in the United States have been especially brilliant in the list of those who have unravelled this problem step by step. It has required clever and painstaking work to devise diets that contained all the necessary constituents for growth except one amino acid, and to handle the animal experiments in such a manner that the results were unequivocal. In the course of these studies W. C. Rose found evidence from his rat responses that there was some previously unknown constituent of hydrolysed casein which was necessary for maintenance, and in searching for it discovered and identified the amino acid threonine. As the result of twenty years of work by all the investigators in the field, Rose finally was able to divide them all into two groups, one comprising the amino acids which must be supplied as such in the food, while the other group can be made by the body and need not be supplied in the protein food.

Essential amino acids
(Must be supplied ready-
made in the diet)
Lysine
Tryptophane
Histidine
Phenylalanine
Leucine
Isoleucine
Threonine
Methionine
Valine
Arginine

$\begin{gathered}\text { Non-essential amino acids } \\ \text { (Need not be supplied in } \\ \text { the diet) }\end{gathered}$
Glycine
Alanine
Serine
Norleucine
Aspartic acid
Glutamic acid
Hydroxyglutamic acid
Proline
Hydroxyproline
Tyrosine
Cystine

The extent to which nitrogen fed in the form of amino acids is synthesized into tissue proteins, both in the form of the fed amino acids and of other amino acids to which the nitrogen is transformed by transamination, has been studied brilliantly by Schoenheimer and his colleagues in the Departments of Chemistry and Physics at Columbia University. They have synthesized amino acids with heavy nitrogen, ${ }^{15} \mathrm{~N}$, have fed the amino acids to rats and mice, and finally have hydrolysed the proteins in their bodies and isolated various amino acids from the hydrolysates. Finally, they have analysed the isolated amino acids for ${ }^{15} \mathrm{~N}$ to determine the amounts of the ingested amino acid that were built into the body proteins, both in the form of the administered amino acid and in the form of other amino acids, formed from the administered one by transamination or other reactions. They have found that incorporation begins almost immediately, and that, while more of the marked ${ }^{15} \mathrm{~N}$ is found still in the amino acid with which it was administered than in any other amino acid, nevertheless a considerable proportion of the marked nitrogen was found distributed.among the other amino acids.

The results of Schoenheimer and his colleagues have entirely dispelled the view that the tissue proteins when once laid down remain as unchanging structural blocks until eventually destroyed by the wear and tear of metabolism. It appears that every protein molecule in the living body is itself alive in the sense that it is continually changing and renewing its structure.

Transmethylation. The discovery of du. Vigneaud that methyl groups can be transferred from methionine to other substances in the body has opened a new field rivalling in interest that of transamination. Du Vigneaud found that some substance in the vitamin B complex could be replaced by methionine. This substance he identified as choline, which is thus identified as one of the necessary vitamins. Du Vigneaud proved that the choline could be formed with the aid of methyl groups from methionine, by administering methionine in which the methyl group was marked by having one of its hydrogens in the form 
of deuterium. When this Iabelled methionine was fed to rats on a choline-free diet, choline could be isolated from their tissues with part of its methyl groups containing the deuterium. It was found that this marked methyl group could be further transferred from the choline to creatine. 'The methyl group of the amino acid methionine could therefore be used by the body in synthesizing two of its essential nonamino acid constituents.

Detoxifying effects of amino acids. We have already noted the manner in which the body uses glycine to combine with and detoxify benzoic acid by forming hippuric acid. In some much less obvious way cystine and methionine protect the liver from intoxication by chloroform. This peculiar effect of the two sulphur-containing amino acids was discovered in 1940 by Miller, Ross, and Whipple. They had observed that a heavy feeding of meat would protect a dog from the effects of a dose of chloroform that would have led to fatal liver degeneration if administered in the fasting state. Investigation of the different types of amino acids yielded by protein digestion proved that only the two containing sulphur had the protective effect.

Concentration of amino acids in the blood plasma and the effect of the endocrines upon it. In the normal fasting adult the concentration of amino acid nitrogen in the plasma is constant at $5 \mathrm{mgm}$. per 100 c.c. If amino acids are absorbed from a protein digest or are injected intravenously there is a transitory rise, and a return soon to the original fasting concentration. The changes in blood amino acid concentration are similar to the changes of blood sugar after administration, orally or by vein, of glucose. There is an equilibrium between the amino acids of the tissues and those of the blood plasma, with the concentration in the tissues ordinarily eight or more times greater than in the plasma. What the forces are that maintain such an unequal balance is not known, but, as in the case of blood glucose, the endocrines have been found to be factors in the control of the plasma concentration. Luck and his collaborators have shown that administration of either insulin or adrenalin lowered the plasma amino acid content of animals. Mirsky has shown that insulin greatly retarded the passage of amino acids from muscles to blood in eviscerated dogs. The effect of insulin on the blood amino acids is much like that on the blood sugar. The opposite effect, to increase the plasma amino acid content, is shown by some of the other hormones. Thus Farr and Alpert in our laboratory have recently found that intravenous administration of the growth. or metabolism-stimulating factors of anterior pituitary extract, of antuitrin, pitressin, or adrenal cortical hormone was followed in dogs by increase of the plasma amino acid content.

Blood amino acid concentrations in disease. From the manner in which hormones can influence the concentration of amino acids in the plasma it is evident that the interpretation of changes found in disease may be difficult. If the plasma amino nitrogen content were merely the mechanical resultant of inflow from the intestines balanced against absorption and metabolism by the tissues, one would anticipate that liver damage would result in a retarded removal of amino acids from the blood, in abnormally large increase in plasma amino acid content during digestion of protein foods, and in abnormally slow return of the plasma amino acids concentration to normal after injections of amino acids. In fact all these results have been reported by various authors, but they seem to be definite only when the hepatic damage has reached a severe grade. This might be expected from the experiments of Frank Mann with partially hepatectomized dogs. He found that it was necessary to remove 80 per cent of the liver before the blood amino acid content began to rise. It does not appear therefore that one can expect blood amino nitrogen determinations to afford a delicate means for detecting moderate degrees of liver injury.

In Bright's disease two extraordinary changes of plasma amino acid content in opposite directions have been observed in our laboratory. One of them was discovered by Kirk in patients in or near uræmic coma. The plasma amino acid nitrogen in some of these patients rose in a day or two from but little above the normal level to six- or eight-fold as much. If, by treatment with saline and glucose injections and other therapeutic measures, recovery from the severely uræmic state could be induced, the plasma amino acid content fell as quickly back to normal. The sudden rise might be explained as due to an accelerated tissue breakdown accompanied by a simultaneous failure of deaminizing function of the liver, but there was no independent evidence that either of these conditions occurred. The mechanism of the amino acid rise in uræmic coma remains a puzzle.

In the opposite direction, Far and MacFayden, using the ninhydrin method first worked out with Dr. Robert Dillon, showed that children with nephrosis are likely to have chronically subnormal plasma amino acid values. But, of particular interest, is the fact that when the patients have the sudden illnesses known as 'nephrotic crises', when sudden collapse occurs without infection or any other obvious cause, the plasma amino acid nitrogen shows precipitate falls to as low as $2 \mathrm{mgm}$. or even $1 \mathrm{mgm}$. per 100 c.c. Whether the cause is an endocrine-influenced shift in the equilibrium between blood and tissues, or some factor that is as yet totally unidentified, remains uncertain. However, regardless of the mechanism of the plasma amino acid fall, the injection of generous amounts of amino acids in the form of predigested milk proteins has regularly produced a marked alleviation of the collapse, and a great reduction in the mortality from these attacks.

Nutrition by intravenously injected amino acids. Shortly after early work cited in this article showed that amino acids were absorbed from the intestine during protein digestion in amounts sufficient to account for all the nitrogen digested, Henriques in Copenhagen demonstrated that nitrogen equilibrium could be maintained with intravenously injected amino acids as the sole intake. He placed a cannula in the neck vein of a goat kept in a stall, and maintained the animal in nitrogen equilibrium for several weeks by giving the great part of the necessary nitrogen in the form of a protein digest hydrolysed completely to amino acids and injected intravenously. The therapeutic application did not follow until twenty years later, when Ellman began the regular use of intravenous injections of predigested casein. He was followed by Farr and MacFadyen, who showed that the injected amino acids were assimilated fully as well as the nitrogen from ingested proteins digested in the alimentary tract. A large part of the nitrogen required can be given in this form over a period of weeks. It appears that this form of nutrition can well supplement intravenous calories in the form of glucose in conditions that prevent adequate feeding by mouth. 